\title{
Potential Energy Curves of Hydrogen Fluoride
}

\section{CASE FILE COPY}

Robert J. FALlon, Joseph T. VANDERSLiCe, and EdWARD A. Mason

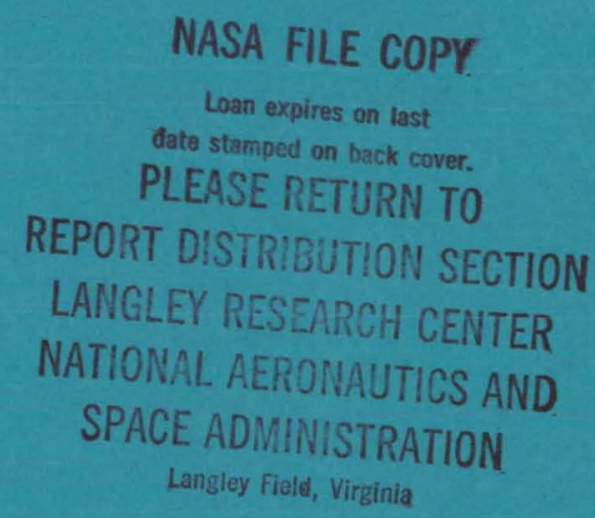

Reprinted from The Journal of Chemical Physics, Vol. 32, No. 3, pp. 698-700, March, 1960 
Reprinted from the Journal of Chemical Physics, Vol. 32, No. 3, 698-700, March, 1960

Printed in U. S. A.

\title{
Potential Energy Curves of Hydrogen Fluoride*
}

\author{
Robert J. Fallon, Joseph T. Vanderslice, and Edward A. Mason \\ Institute for Molecular Physics, Universily of Maryland, College Park, Maryland
}

(Received November 2, 1959)

\begin{abstract}
Potential energy curves for the $X^{1 \Sigma^{+}}$and $V^{1 \Sigma^{+}}$.states of HF and DF have been calculated by the Rydberg-Klein-Rees method. The results calculated from the different sets of data for HF and DF are found to be in very good agreement. The theoretical results of Karo are compared to the experimental results obtained here.
\end{abstract}

\section{INTRODUCTION}

$\mathbf{Q}^{\mathrm{T}}$ UANTUM mechanical calculations on molecular systems are known to give reliable estimates of the total energy of such systems, but, in general, the values calculated for the binding energies of diatomic molecules are in poor agreement with experiment. The reason for this is that the error in the total energy, although small on a percentage basis, is often of the same order of magnitude as the binding energy. In relatively simple cases, such as $\mathrm{H}_{2}$, the problem can be handled by brute force methods. ${ }^{1}$ For more complicated systems containing a large number of electrons, such techniques are as yet out of the question. It would seem that approximation schemes are the most desirable at the present time if one wants to be able to calculate accurate potential energy curves for diatomic molecules.

In order to test the reliability of such approximation schemes, it is well to have reliable potential energy curves for diatomic systems against which the various schemes can be tested. Since a relatively large amount of effort has been expanded by theoreticians on the hydrogen fluoride molecule, ${ }^{2-8}$ it was felt that it would be worthwhile to have an accurate potential curve for this system.

Recently, Johns and Barrow ${ }^{9}$ have obtained some excellent spectroscopic data on $\mathrm{HF}$ and $\mathrm{DF}$, and these, together with older data, ${ }^{10-12}$ have enabled us to obtain

\footnotetext{
* This research was supported in part by the National Aeronautics and Space Administration.

1 W. Kolos and C. C. J. Roothaan, paper presented at the International Conference on Molecular Quantum Mechanics, Boulder, Colorado, June 7-15, 1959 (to be published in Revs. Modern Phys.).

2 A. B. F. Duncan, J. Am. Chem. Soc. 77, 2107 (1955).

${ }^{3}$ D. Kastler, J. chim. phys. 50, 556 (1953).

A. C. Hurley, Proc. Roy. Soc. (London) A248, 119 (1958).

${ }^{5}$ M. Krauss, J. Chem. Phys. 28, 1021 (1958).

6 A. M. Karo and L. C. Allen, J. Am. Chem. Soc. 80, 4496 (1958).

${ }_{7}^{7}$ A. M. Karo, Quarterly Progress Report; Solid State and Molecular Theory Group, M. I. T., October 15, 1957, p. 16; A. M.

Karo and L. C. Alllen, J. Chem. Phys. 31, 968 (1959).

${ }^{8}$ R. A. Ballinger, Mol. Phys. 2, 139 (1959).

J. W. C. Johns and R. F. Barrow, Proc. Roy. Soc. (London) A251, 504 (1959)

${ }_{10}$ Talley, Kaylor, and Nielsen, Phys. Rev. 77, 529 (1950).

"I S. M. Naudé and H. Verleger, Proc. Phys. Soc. (London) A63, 470 (1950).

12 Kuipers, Smith, and Nielsen J. Chem. Phys. 25, 275 (1956)
}

experimental potential energy curves for the $X^{1} \Sigma^{+}$ and $V^{t} \Sigma^{+}$states of these molecules over a range of internuclear distance. The actual curves were calculated by the Rydberg-Klein-Rees (RKR) method. ${ }^{13-16}$

The experimental curve for the $X^{1} \Sigma^{+}$state was compared with a Morse curve and with one obtained theoretically by Karo. ${ }^{7}$

\section{RYDBERG-KLEIN-REES METHOD AND SPECTROSCOPIC DATA}

The RKR method is a semiclassical procedure for obtaining the potential energy curves for diatomic molecules. The curves obtained with this method are based directly on the measured vibrational and rotational energy levels of the molecule and do not depend on the assumption of any empirical potential form. The method has been adequately described in recent $\operatorname{articles}^{15-16}$ so that we shall omit the details of the procedure. It suffices to say that the method is quite rapid and appears to give reliable results.

The spectroscopic data for the $X^{1} \Sigma^{+}$states of $\mathrm{HF}$ and DF do not include all the vibrational levels from the lowest to the highest. Johns and Barrow ${ }^{9}$ have obtained data for the higher levels, whereas Nielsen and co-workers, ${ }^{10,12}$ and Naudé and Verleger ${ }^{11}$ have obtained data on the lower. Johns and Barrow ${ }^{9}$ have carried out. what appears to be a reliable interpolation between the upper and the lower levels so that the intermediate levels could be inferred from the standard spectroscopic formulas. We have used the observed data plus results obtained by the interpolation method to calculate experimental curves for the $X^{1} \Sigma^{+}$state of both systems.

\section{RESULTS AND DISCUSSION}

The results for the $X^{1} \Sigma^{+}$and $V^{1} \Sigma^{+}$states of $\mathrm{HF}$ and DF are listed in Tables I to IV and are shown as solid lines in Fig. 1. The corresponding curves for the two systems do not differ by more than $\pm 0.001 \mathrm{~A}$. This indicates that the different sets of data and the inter-

${ }^{13}$ R. Rydberg, Z. Physik 73, 376 (1931); 80, 514 (1933).

${ }^{14}$ O. Klein, Z. Physik 76, 226 (1932).

${ }^{15}$ A. L. G. Rees, Proc. Phys. Soc. (London) 59, 998 (1947)

${ }^{16}$ Vanderslice, Mason, Maisch, and Lippincott, J. Mol. Spectroscopy 3, 17 (1959); Vanderslice, Mason, and Lippincott, J. Chem. Phys. 30, 129 (1959); Vanderslice, Mason, and Maisch, ibid. 31, 738 (1959). 
TABLE I. Potential energy of the $X^{1} \Sigma^{+}$state of HF.

\begin{tabular}{rcccc}
\hline$v$ & $V\left(\mathrm{~cm}^{-1}\right)$ & $V(\mathrm{ev})$ & $r_{\max }(\mathrm{A})$ & $r_{\min }(\mathrm{A})$ \\
\hline 0 & 2047 & 0.2537 & 1.023 & 0.836 \\
1 & 6008 & 0.7448 & 1.115 & 0.786 \\
2 & 9798 & 1.215 & 1.187 & 0.755 \\
3 & 13419 & 1.663 & 1.253 & 0.733 \\
4 & 16878 & 2.092 & 1.317 & 0.716 \\
5 & $(20193)$ & $(2.503)$ & 1.379 & 0.701 \\
6 & $(23351)$ & $(2.895)$ & 1.441 & 0.689 \\
7 & $(26350)$ & $(3.266)$ & 1.503 & 0.679 \\
8 & $(29191)$ & $(3.619)$ & 1.567 & 0.670 \\
9 & $(31875)$ & $(3.951)$ & 1.633 & 0.662 \\
10 & 34335 & 4.256 & 1.701 & 0.656 \\
11 & 36705 & 4.550 & 1.772 & 0.650 \\
12 & 38921 & 4.825 & 1.847 & 0.644 \\
13 & 40973 & 5.079 & 1.928 & 0.639 \\
14 & 42850 & 5.312 & 2.018 & 0.633 \\
15 & 44543 & 5.522 & 2.121 & 0.630 \\
16 & 46031 & 5.706 & 2.244 & 0.626 \\
17 & 47292 & 5.862 & 2.397 & 0.623 \\
18 & 48294 & 5.987 & 2.604 & 0.621 \\
\hline \hline
\end{tabular}

a Numbers in parentheses are interpolated values.

TABLE II. Potential energy of the $X^{1 \Sigma^{+}}$state of DF.

\begin{tabular}{|c|c|c|c|c|}
\hline$v$ & $V\left(\mathrm{~cm}^{-1}\right)$ & $V(\mathrm{ev})$ & $r_{\max }(\mathrm{A})$ & $r_{\min }(\mathrm{A})$ \\
\hline 0 & 1488 & 0.1845 & 1.005 & 0.847 \\
\hline 1 & 4395 & 0.5448 & 1.079 & 0.802 \\
\hline 2 & 7211 & 0.8938 & 1.137 & 0.774 \\
\hline 3 & (9 947) & $(1.233)$ & 1.188 & 0.753 \\
\hline 4 & $(12600)$ & $(1.562)$ & 1.236 & 0.737 \\
\hline 5 & $\left(\begin{array}{ll}15 & 169\end{array}\right)$ & $(1.880)$ & 1.283 & 0.723 \\
\hline 6 & $(17655)$ & $(2.189)$ & 1.328 & 0.711 \\
\hline 7 & $\left(\begin{array}{lll}20 & 058\end{array}\right)$ & $(2.486)$ & 1.373 & 0.701 \\
\hline 8 & $\left(\begin{array}{lll}22 & 377\end{array}\right)$ & $(2.774)$ & 1.418 & 0.692 \\
\hline 9 & $(24613)$ & (3.051) & 1.462 & 0.684 \\
\hline 10 & $(26766)$ & $(3.318)$ & 1.508 & 0.677 \\
\hline 11 & $\left(\begin{array}{lll}28 & 834\end{array}\right)$ & $(3.574)$ & 1.554 & 0.670 \\
\hline 12 & $(30820)$ & $(3.820)$ & 1.601 & 0.664 \\
\hline 13 & $(32722)$ & $(4.056)$ & 1.649 & 0.659 \\
\hline 14 & $(34541)$ & $(4.282)$ & 1.699 & 0.654 \\
\hline 15 & $\left(\begin{array}{lll}36 & 276\end{array}\right)$ & $(4.497)$ & 1.750 & 0.650 \\
\hline 16 & 37815 & 4.688 & 1.803 & 0.646 \\
\hline 17 & 39385 & 4.882 & 1.858 & 0.642 \\
\hline 18 & 40866 & 5.066 & 1.918 & 0.638 \\
\hline 19 & 42257 & 5.238 & 1.982 & 0.635 \\
\hline 20 & 43552 & 5.399 & 2.051 & 0.632 \\
\hline 21 & 44746 & 5.547 & 2.129 & 0.630 \\
\hline 22 & 45832 & 5.681 & 2.216 & 0.627 \\
\hline 23 & 46799 & 5.801 & 2.315 & 0.624 \\
\hline
\end{tabular}

- Numbers in parentheses are interpolated values.

TABLE III. Potential energy of the $V^{1} \Sigma^{+}$state of HF.

\begin{tabular}{cccccc}
\hline$v$ & $V\left(\mathrm{~cm}^{-1}\right)$ & $V(\mathrm{ev})$ & $T_{\varepsilon}+V(\mathrm{ev})$ & $r_{\max }(\mathrm{A})$ & $r_{\min }(\mathrm{A})$ \\
\hline 0 & 574.8 & 0.0713 & 10.5813 & 2.275 & 1.924 \\
1 & 1699 & 0.2106 & 10.7206 & 2.426 & 1.811 \\
2 & 2788 & 0.3456 & 10.8556 & 2.540 & 1.735 \\
3 & 3844 & 0.4764 & 10.9864 & 2.641 & 1.675 \\
4 & 4868 & 0.6035 & 11.1135 & 2.733 & 1.625 \\
5 & 5862 & 0.7266 & 11.2366 & 2.822 & 1.580 \\
\hline
\end{tabular}

a $T_{e}$ is the energy difference between the bottom of the potential curve in question and the bottom of the curve for the $X{ }^{1} \Sigma^{+}$state. The value of $T_{e}$ used is $10.5100 \mathrm{ev}$. The value appears to be uncertain to $\pm 0.005 \mathrm{ev}$.
TABLE IV. Potential energy of the $V^{1} \Sigma^{+}$state of DF.

\begin{tabular}{cccccc}
\hline \hline$v$ & $V\left(\mathrm{~cm}^{-1}\right)$ & $V(\mathrm{ev})$ & $T_{\text {o }}+V(\mathrm{ev})$ & $\boldsymbol{r}_{\max }(\mathrm{A})$ & $\boldsymbol{r}_{\min }(\mathrm{A})$ \\
\hline 0 & 417.4 & 0.0517 & 10.5617 & 2.244 & 1.946 \\
1 & 1239 & 0.1536 & 10.6636 & 2.369 & 1.848 \\
2 & 2042 & 0.2532 & 10.7632 & 2.462 & 1.782 \\
3 & 2828 & 0.3506 & 10.8606 & 2.542 & 1.730 \\
\hline
\end{tabular}

polations are consistent and also that the RKR method is capable of this precision.

The heavy dashed line in Fig. 1 represents a Morse curve with constants derived from the spectroscopic data. The agreement with the experimental curve is surprisingly good in view of the fact that the Morse curve is often a rather poor representation of the actual curves for the cases where it has been compared with the experimental curves. ${ }^{16}$

The light dashed line in Fig. 1 is the curve obtained by Karo for the $X^{1} \Sigma^{+}$state using an LCAO-MO method which included a large amount of configuration interaction. Although the calculations of Karo are quite extensive, it can not be said that the results are in too good agreement with the actual experimental curve. Karo, of course, was aware of this in his original work. ${ }^{7}$

In view of the difficulties inherent in an $a b$ initio calculation on many-electron systems, various approximation schemes have been suggested in the past ${ }^{17}$

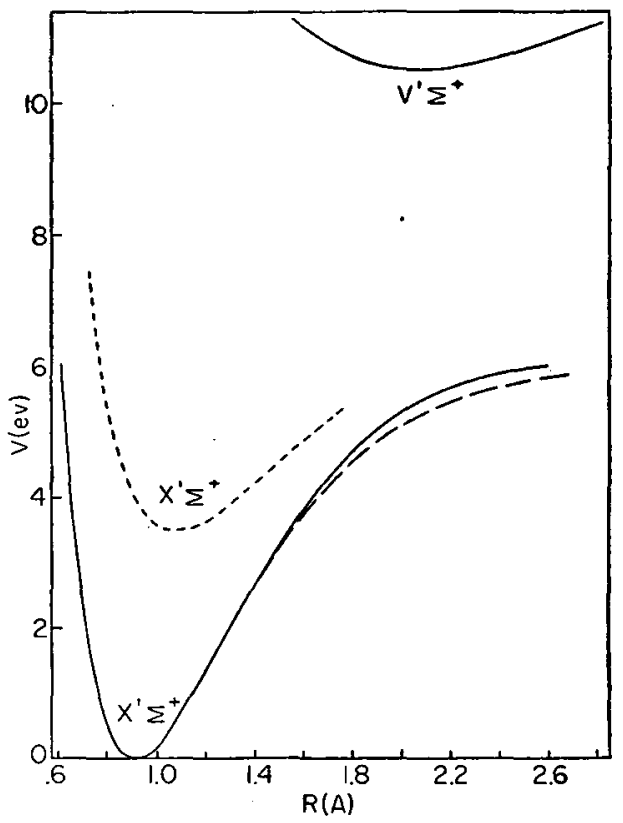

FIg. 1. Potential energy curves for HF and DF. The solid lines are curves derived directly from the spectroscopic data by the RKR method. The heavy dashed line represents a Morse curve and the light dashed line corresponds to the potential curve obtained theoretically by Karo.

${ }^{17}$ Eyring, Walter, and Kimball, Quantum Chemistry (John Wiley \& Sons, Inc., New York, 1944). 
for the calculation of interaction energies and un- hydrogen fluoride, as well as for other systems, ${ }^{16,16}$ it doubtedly others will be put forward in the future. ${ }^{18}$ is now possible to check the adequacy of approximation With a knowledge of the experimental curves for schemes which may be proposed.

\section{ACKNOWLEDGMENT}

${ }^{18} \mathrm{H}$. Preuss, Technical Note No. 24 from the Quantum Chemistry Group, Uppsala University, Uppsala, Sweden, April 20, 1959.

The authors are grateful to Louis T. Ho for checking some of the calculations. 